\title{
CBP80-Promoted mRNP Rearrangements during the Pioneer Round of Translation, Nonsense-Mediated mRNA Decay, and Thereafter
}

\author{
L.E. Maquat, J. Hwang, H. SATO, AND Y. TANG \\ Department of Biochemistry and Biophysics, The Center for RNA Biology, School of Medicine and Dentistry, \\ University of Rochester, Rochester, New York 14642 \\ Correspondence:lynne_maquat@urmc.rochester.edu
}

\begin{abstract}
In mammalian cells, two different messenger ribonucleoproteins (mRNPs) serve as templates for protein synthesis. Newly synthesized mRNPs bound by the cap-binding protein heterodimer CBP80-CBP20 (CBC) initially undergo a pioneer round of translation. One purpose of this round of translation is to ensure the quality of gene expression, as exemplified by nonsense-mediated messenger RNA (mRNA) decay (NMD). NMD largely functions to eliminate mRNAs that prematurely terminate translation, although NMD also contributes to proper gene control, and it targets CBC-bound mRNPs. CBC-bound mRNPs are remodeled to eukaryotic translation initiation factor (eIF)4E-bound mRNPs in steps that (1) are a consequence of the pioneer round of translation and (2) occur independently of translation. Rather than supporting NMD, eIF4E-bound mRNPs provide for the bulk of cellular protein synthesis and are the primary targets of mRNA decay mechanisms that conditionally regulate gene expression. Here, we overview cellular processes by which CBC-bound mRNPs are remodeled to eIF4E-bound mRNPs. We also describe the molecular movements of certain factors during NMD in view of the influential role of CBP80.
\end{abstract}

mRNAs in eukaryotic cells shed acond acquire proteins throughout their life span while undergoing modification, transport, translation, and decay. The remodeling of mRNPs occurs within nuclei, on both sides of the nuclear pore complex during the process of mRNP export from the nucleus to the cytoplasm, and within the cytoplasm (see, e.g., Tran et al. 2007 and references therein). Proper remodeling is critical to the fidelity of gene expression. Remodeling could, in theory, be promoted passively, e.g., by differences in the concentration of a particular protein at different sites of the cell, or actively, e.g., in reactions that involve energy-dependent helicases or conformational changes induced by the binding of one or more additional proteins.

In mammalian cells, newly synthesized mRNAs, which support the pioneer round of translation, can be distinguished from steady-state mRNAs, which support the bulk of cellular translation, by virtue of their cap-binding protein(s) (for recent review, see Maquat et al. 2010). Data indicate that the cap-binding heterodimer $\mathrm{CBC}$ binds to newly synthesized pre-mRNAs within nuclei and, after premRNA processing, shuttles to the cytoplasm in association with mRNA to support the first round of translation, which we call the pioneer round of translation (Visa et al. 1996; Ishigaki et al. 2001; Lejeune et al. 2002; Chiu et al. 2004; Cheng et al. 2006; Sato et al. 2008). The finding that CBCbound mRNAs, which in mammalian cells are precursors to eIF4E-bound mRNAs, associate with polysomes as do their eIF4E-bound counterparts is consistent with the idea that the replacement of $\mathrm{CBC}$ by eIF4E occurs on polysomes (Stephenson and Maquat 1996; Chiu et al. 2004; Sato et al. 2008 and references therein). Exon-junction complexes (EJCs) are deposited onto newly synthesized mRNAs as a consequence of pre-mRNA splicing (LykkeAndersen et al. 2000, 2001; Kim et al. 2001; Lejeune et al. 2002; Chamieh et al. 2008) and detectable on spliced CBCbound mRNAs but not on spliced eIF4E-bound mRNAs (Ishigaki et al. 2001; Lejeune et al. 2002; Hosoda et al. 2005; Kashima et al. 2006). Like CBC, the NMD factors UPF2 and UPF3X (also called UPF3b), which are stable constituents of EJCs, co-sediment with polysomes (Chiu et al. 2004). It follows that EJCs are also removed while they are associated with polysomes, consistent with their cytoplasmic role during NMD (Singh et al. 2007).

Steps of CBC-bound mRNP remodeling also occur during the process of NMD. In mammalian cells, NMD is a consequence of nonsense-codon recognition during a pioneer round of translation in a mechanism that generally involves an EJC situated more than $\sim 25-35$ nucleotides downstream from a premature termination codon (PTC) (for recent reviews, see Isken and Maquat 2008; Rebbapragada and Lykke-Andersen 2009; Silva and Romão 2009; Nicholson et al. 2010). When translation terminates at a PTC that is followed by an appropriately positioned EJC, SURF forms at the PTC. SURF consists of the SMG1 phosphoinositide 3-kinase-related protein kinase, the UPF1 NMD factor, and the two translation termination factors referred to as eukaryotic release factor $(\mathrm{e} R F) 1$ and e $R F 3$ (Kashima et al. 2006; Yamashita et al. 2009). After SURF formation, all (Yamashita et al. 2009) or, possibly more likely, part (Kashima et al. 2006; Hwang et al. 2010) of SURF joins the downstream EJC (see below). SMG1 then mediates the phosphorylation of UPF1 (Kashima et al. 2006; Wittmann et al. 2006; Yamashita et al. 2009). Phos- 
phorylated UPF1 subsequently binds eIF3 of the preinitiation complex that is poised at the translation initiation codon to suppress further translation of the NMD targeta step that data indicate is critical for mRNA decay (Isken et al. 2008). Data also reveal that phosphorylated UPF1 recruits nucleases that mediate mRNA decay from both $5^{\prime}$ and $3^{\prime}$ ends as well as endonucleotypically (Isken et al. 2008; Franks et al. 2010 and references therein).

We set out to determine how CBC-bound mRNP is remodeled to eIF4E-bound mRNP with the idea that the pioneer round of translation itself could activate mRNP remodeling. We found that $\mathrm{CBP} 80$ of $\mathrm{CBC}$ has a crucial role in some steps of $\mathrm{mRNP}$ remodeling because of its function as a translation initiation factor. For example, CBP80 supports the displacement of EJCs by elongating ribosomes during the pioneer round of translation (Sato and Maquat 2009). Remarkably, however, CBP80 promotes the replacement of CBC by eIF4E at mRNA caps independent of the pioneer round of translation but dependent on its interaction with the adapter protein importin $\alpha$ (IMP $\alpha$ ) in a way that relies on the IMP $\alpha$-mediated recruitment of the karyopherin IMP $\beta$ (Dias et al. 2009; Sato and Maquat 2009). Given that CBP80 also interacts directly with the NMD factor UPF1 and promotes the binding of UPF1 to UPF2 in cells and in vitro (Hosoda et al. 2005), we additionally examined the role of CBP80 in SURF complex formation as well as the joining of UPF1 to a PTC-distal EJC. Results reveal that the interaction of CBP80 with UPF1 indeed promotes the joining of SMG1 and UPF1 first to the eRF1-eRF3 heterodimer at a PTC and subsequently to a PTC-distal EJC (Hwang et al. 2010).

\section{SECTION THEMES}

\section{CBP80-Supported Pioneer Round of Translation Promotes Removal of EJC Constituents and Replacement of PABPN1 by PABPC1}

CBP80 and CBP20 associate with cap structures as a heterodimer rather than individually: CBP20, like its steady-state counterpart eIF4E, envelops the 7-methylguanine of the cap structure between two amino acid side chains (Marcotrigiano et al. 1997; Matsuo et al. 1997; Niedzwiecka et al. 2002; Tomoo et al. 2002). Unlike eIF4E, however, the binding of CBP20 to an mRNA cap requires CBP80 for stability (Izaurralde et al. 1994; Mazza et al. 2001, 2002; Calero et al. 2002). Cap-bound CBC functions analogously to cap-bound eIF4E as a translation initiation factor: Both interact directly with eIF4G, which serves as a platform for the loading of translation initiation factors that include eIF3 and the major cytoplasmic poly(A)-binding protein PABPC1, and both recruit the 40S ribosomal subunit-containing preinitiation complex to mRNA (Chiu et al. 2004; Lejeune et al. 2004; Isken et al. 2008; Sonenberg and Hinnebusch 2009).

At least five lines of evidence indicate that the CBCpromoted pioneer round of translation augments the removal of postsplicing EJCs (Fig. 1). First, NMD fails to occur if translation terminates either less than $~ 25-35 \mathrm{nu}-$ cleotides upstream of all EJCs or downstream from all EJCs, both of which would result in the ribosome-mediated displacement of all EJCs considering that 25-35 nucleotides approximate the closest distance that the leading edge of the terminating ribosome can be situated relative

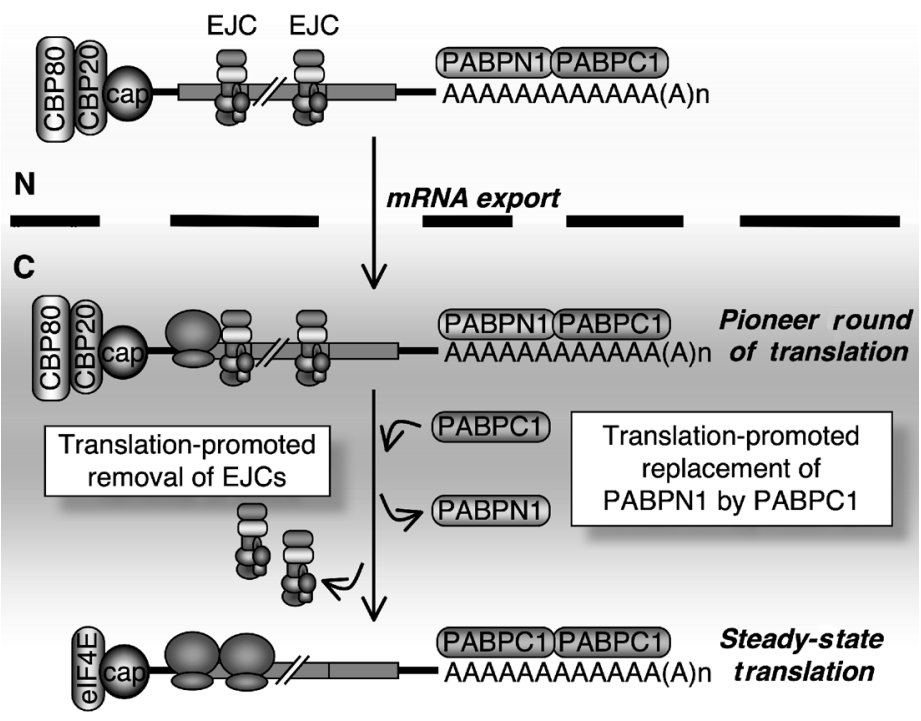

Figure 1. Pioneer round of translation promotes the removal of EJC constituents and replacement of PABPN1 by PABPC1. Pioneer translation initiation complex is exported from the nucleus to the cytoplasm bound by (1) CBC, which consists of CBP80-CBP20 at the $5^{\prime}$ cap and functions not only in the export of spliced mRNA but also as a translation initiation factor; (2) EJCs, which generally contain eIF4AIII and the NMD factors UPF2 and UPF3X or UPF3, located 20-25 nucleotides upstream of exon-exon junctions if the mRNA underwent splicing; and (3) PABPN1 and PABPC1 at the poly(A) tail. Other factors that typify the pioneer translation initiation complex include the eIF3 and eIF4G factors and IMP $\alpha$ (not shown; see Fig. 2). Data indicate that the pioneer round of translation promotes the removal of the EJCs and the replacement of PABPN1 by PABPC1. EJC removal is mediated by elongating ribosomes, presumably in association with PYM (not shown), which explains why a PTC situated less than $~ 30-35$ nucleotides upstream of or downstream from the 3'-most EJC generally does not trigger NMD: There is no surviving EJC downstream from the PTC (see Fig. 3). The mechanism by which translation promotes the replacement of PABPN1 by PABPC1 is less certain. (N) nucleus, (C) cytoplasm. 
to an EJC before physically contacting the EJC (Isken and Maquat 2007; see below). Second, mRNA generated by splicing in cell-free extracts coimmunoprecipitates with the Y14 EJC constituent after incubation in translationally active extracts if the $5^{\prime}$-untranslated region (UTR) harbors a higher-order structure that blocks the translation initiation codon (Dostie and Dreyfuss 2002). Third, spliced mRNA coimmunoprecipitates with Y14 after purification from cytoplasmic lysates of HEK293T if the 5'UTR of the mRNA harbors a higher-order structure that blocks translation initiation (Dostie and Dreyfuss 2002). Fourth, inhibiting recruitment of the preinitiation complex to an AUG translation initiation codon that resides downstream from an iron response element (IRE) by decreasing the level of intracellular iron increases the levels of UPF3X and eIF4AIII EJC constituents that coimmunoprecipitate with the IRE-containing mRNA (Sato and Maquat 2009). In particular, analyses of cytoplasmic lysates that, unlike total-cell lysates, contain a significant fraction of EJCbound mRNA that is accessible to the translational machinery, revealed a larger increase in the amount of EJC-bound IRE-containing mRNA in cells depleted of iron relative to cells replete of iron (Sato and Maquat 2009). It is likely that EJC constituents other than those specifically studied in these reports are also removed by translating ribosomes, especially considering that one of the constituents studied - eIF4AIII - anchors the EJC to mRNA (Le Hir and Andersen 2008). Fifth, ribosomebound PYM, like overexpressed PYM, promotes the removal of EJCs from spliced mRNA, implicating the involvement of not only translation-dependent but possibly also translation-independent mechanisms of EJC removal, contingent upon how much cytoplasmic PYM is free of ribosomes and can bind to EJCs (Gehring et al. 2009).

The CBC-promoted pioneer round of translation also augments the replacement of the largely nuclear poly(A)binding protein PABPN1 by PABPC1. Both PABPs are present on CBC-bound mRNA, with which they are transported from nuclei to the cytoplasm (Fig. 1) (Afonina et al. 1998; Ishigaki et al. 2001; Bear et al. 2003; Chiu et al. 2004; Hosoda et al. 2006). Inhibiting the recruitment of the preinitiation complex to an AUG translation initiation codon that resides downstream from the aforementioned IRE increases the level of PABPN1 and decreases the level of PABPC1 that coimmunoprecipitate with IRE-containing mRNA (Sato and Maquat 2009). As with EJC loss, the replacement of PABPN1 by PABPC1 is more dramatic using cytoplasmic lysates compared with total-cell lysates, consistent with the idea that translation promotes these steps of mRNP remodeling (Sato and Maquat 2009).

Although data indicate that the pioneer round of translation promotes the loss of EJCs by physically displacing the EJCs, how this round of translation augments the replacement of PABPN1 by PABPC1 is less certain. Replacement might occur during translation termination, when PABPC1 is thought to interact directly with eRF3 (Hoshino et al. 1999) via a region that is not conserved in PABPN1 (Kühn and Wahle 2004).

\section{Binding of Karyopherin IMP $\beta$ to CBP80-Bound IMP $\alpha$, Rather than the Pioneer Round of Translation, Promotes the Replacement of CBC by eIF4E at mRNA Caps}

CBP80 also promotes the replacement of $\mathrm{CBC}$ by eIF4E; however, replacement is not augmented by the pioneer round of translation because inhibiting preinitiationcomplex recruitment to an AUG translation initiation codon is of no consequence to the amount of either CBP80 or eIF4E that coimmunoprecipitates with IRE-containing mRNA (Sato and Maquat 2009). A priori, this finding is remarkable because if eIF4E were to replace $\mathrm{CBC}$ before the pioneer round of translation, PTC-containing mRNA would be immune to NMD given the critical role of CBP80 in NMD. The replacement of CBC by eIF4E at an mRNA cap appears to involve transitioning from a more tightly bound complex to a more loosely bound complex according to in vitro-binding studies of CBC and eIF4E to mononucleotide, dinucleotide, and tetranucleotide $5^{\prime}$ cap analogs (Worch et al. 2005).

On the basis of a number of findings, transitioning is, at least in part, accomplished by the binding of the karyopherin IMP $\beta$ to CBC-bound IMP $\alpha$ to promote the dissociation of $\mathrm{CBC}$ from newly exported mRNAs, independent of translation, and augment the binding of eIF4E to the resulting unoccupied $5^{\prime}$ caps (Fig. 2). First, binding of IMP $\alpha$ directly to the bipartite nuclear localization signal of CBP80 is remarkably resistant to high salt concentrations and is not dissociated within Xenopus oocyte nuclei (Görlich et al. 1996; Lewis and Izaurralde 1997). Second, IMP $\alpha$ coimmunoprecipitates with the $5^{\prime}$ end of mammalian-cell mRNAs in both nuclear and cytoplasmic fractions, independent of translation (Sato and Maquat 2009). Third, IMP $\alpha$ coimmunoprecipitates with mammalian-cell CBP80 in an RNase A-resistant manner, consistent with the interaction being direct (Sato and Maquat 2009). Fourth, inhibiting the interaction of IMP $\alpha$ with IMP $\beta$ by expressing (1) the 41 -amino-acid IMP $\beta$ binding domain of IMP $\alpha$ or (2) RAN(Q69L), which is a variant of the RAS-related small guanine nucleotide-binding protein RAN that binds but cannot hydrolyze GTP, results in an increase in the level of CBP80-bound mRNA and a concomitant decrease in the level of eIF4E-bound mRNA (Sato and Maquat 2009). Normally, after IMP $\beta$ removes CBC-IMP $\alpha$ from mRNA caps, the CBC-IMP $\alpha$ IMP $\beta$ heterotrimer is subsequently transported back to the nucleus where CBC-IMP $\alpha$ is free to reassociate with the caps of newly synthesized transcripts that are generally destined for the cytoplasm, and IMP $\beta$ is separately exported to the cytoplasm as a complex with RAN-GTP (Fig. 2). Fifth, based on (1) an X-ray structure of an IMP $\alpha$ CBC complex, (2) a model of a CBC-IMP $\alpha$-IMP $\beta$ complex, and (3) biochemical and mutagenesis data, IMP $\beta$ was formulated to bind not only the IMP $\beta$-binding domain (IBB) of IMP $\alpha$, but also the carboxy-terminal region of CBP20 to weaken the affinity of CBP20 for capped RNA and promote the release of CBC (Dias et al. 2009). Interestingly, IMP $\alpha$ was additionally shown to bind cap methyltransferase in vitro and stimulate the methylation 


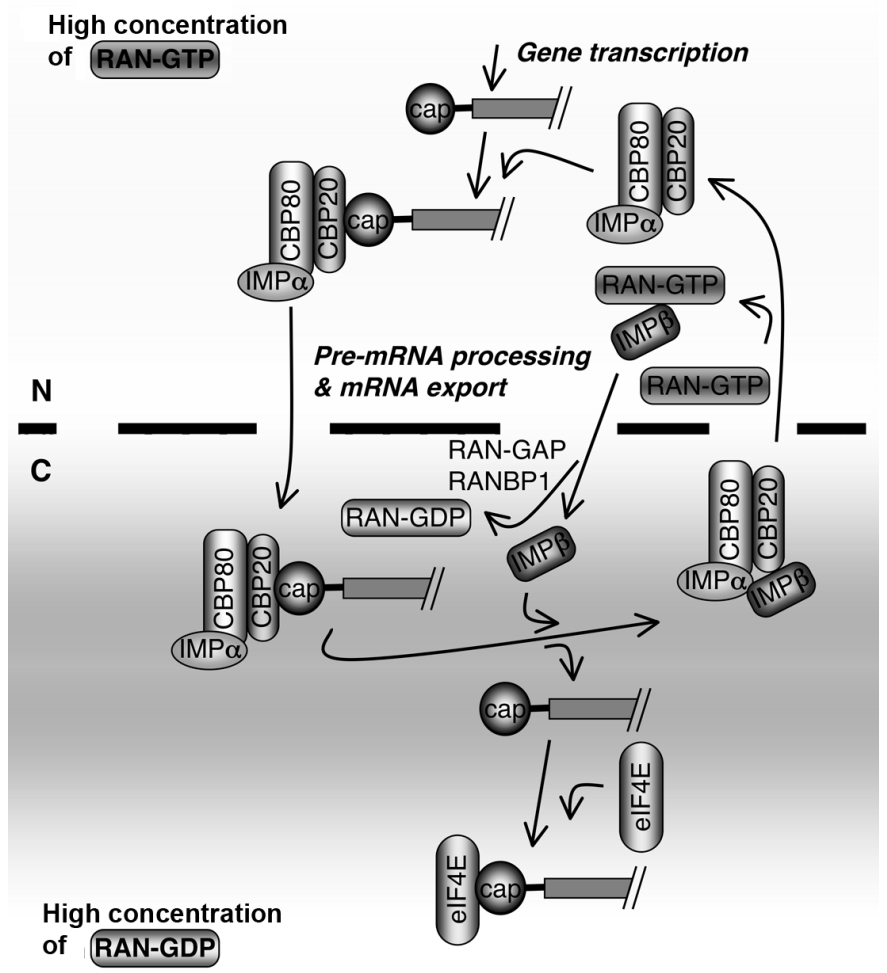

Figure 2. Binding of karyopherin IMP $\beta$ to CBP80bound IMP $\alpha$, rather than the pioneer round of translation, promotes replacement of CBP80-CBP20 (CBC) by eIF4E at mRNA caps, as in Figure 1. However, here, IMP $\alpha$ is shown to be a constituent of CBC by virtue of its direct contact with CBP80. IMP $\alpha$ binds the bipartite nuclear localization signal of CBP80 on unspliced pre-RNA, possibly concomitantly with CBC binding to nascent transcripts. The pioneer round of translation has no detectable effects on either the association of IMP $\alpha$ with CBC or replacement of CBC by eIF4E. Instead, the interaction of the karyopherin IMP $\beta$ with IMP $\alpha$ in the cytoplasm promotes replacement of CBC by eIF4E. Replacement involves RANGTP-mediated delivery of nuclear IMP $\beta$ to the cytoplasm. GTPase-activating protein RAN-GAP together with RAN-binding protein (RANBP) 1 converts RAN-GTP-IMP $\beta$ to RAN-GDP and IMP $\beta$ to promote IMP $\beta$ binding to IMP $\alpha-C B C$ (Dias et al. 2009; Sato and Maquat 2009). Replacement of CBC by eIF4E is followed by the movement of IMP $\beta$-IMP $\alpha-C B C$ to nuclei. Once in the nucleus, IMP $\beta$ dissociates from IMP $\alpha-C B C$. This frees IMP $\alpha-C B C$ to bind to the $5^{\prime}$ cap of newly made transcripts to function again in nuclear export and the cytoplasmic remodeling of mRNP. of in vitro-synthesized GpppG-RNA (Wen and Shatkin 2000). This indicates that IMP $\alpha$ begins to function very early in mRNA biogenesis, consistent with its coimmunoprecipitating with unspliced pre-mRNA (Sato and Maquat 2009)

In theory, replacement of $\mathrm{CBC}$ by eIF4E might also involve mass action considering that the level of eIF4E relative to $\mathrm{CBC}$ is higher in the cytoplasm than in nuclei (Sato and Maquat 2009). Moreover, it has been suggested that replacement could be mediated by specific interactions among eIF4E, CBC, and eIF4G that decrease the affinity of CBC for the cap (Fortes et al. 2000; McKendrick et al. 2001). Nevertheless, although the binding of eIF4G to eIF4E indeed strengthens the interaction between eIF4E and the mRNA cap (Haghighat and Sonenberg 1997; Gross et al. 2003), eIF4G could also conceivably stabilize the interaction between $\mathrm{CBC}$ and the cap because eIF4G function appears to extend to the pioneer round of translation (Lejeune et al. 2004).

\section{During NMD, Interaction of CBP80 with UPF1 Promotes SMG1 and UPF1 Joining with eRF1 and eRF3 at a PTC to Form SURF and the Subsequent Binding of SMG1 and UPF1 to a PTC-Distal EJC}

Because CBP80 promotes the pioneer round of translation, it follows that CBP80 would augment the efficiency of NMD. However, the role of CBP80 in NMD extends well beyond its capacity as a translation initiation factor. CBP80 interacts directly but transiently or weakly with UPF1 (Hosoda et al. 2005; Hwang et al. 2010), and expressing either the CBP80-binding domain of UPF1 (i.e., UPF1[419-700]) or the UPF1-binding domain of CBP80 (i.e., CBP80[664-790]) to specifically inhibit the interaction between UPF 1 and CBP80 hinders at least two steps during the process of NMD (Fig. 3) (Hwang et al. 2010). One step is the formation of SURF at a PTC, which as noted above is critical for PTC recognition. In support of this, UPF1(419-700) as well as CBP80(664-790) inhibit the coimmunoprecipitation of SMG1 and UPF1, presumably as a heterodimer, with eRF1 and eRF3, which themselves form a heterodimer. Another step is the binding of SMG1 and UPF1, presumably as a heterodimer that derives from SURF, to an EJC. We find that neither eRF1 nor eRF3 detectably accompanies SMG1 and UPF1 to the EJC. Data also indicate that PTC-containing transcripts coimmunoprecipitate $\sim 10$-fold to 20 -fold more efficiently with UPF1 than do their PTC-free counterparts (Hwang et al. 2010). This PTC-enhanced binding of UPF1 to mRNA is augmented by the interaction between UPF 1 and CBP80 and localizes to the PTC and/or downstream EJC rather than to the CBC-bound mRNA cap.

All of these findings indicate that UPF1 binding to mRNA during the process of NMD is far from static (Fig. 3 ). Data suggest that UPF1 transiently or weakly binds to the $\mathrm{CBC}$ of newly synthesized mRNA before or in early stages of the pioneer round of translation. In the case of mRNAs that are targeted for NMD, SURF forms following termination of the pioneer round of translation. SURF formation occurs before SMG1 and UPF1 associate with an EJC because coimmunoprecipitation of SMG1 with UPF1, eRF1, and eRF3 increases if the cellular concen- 

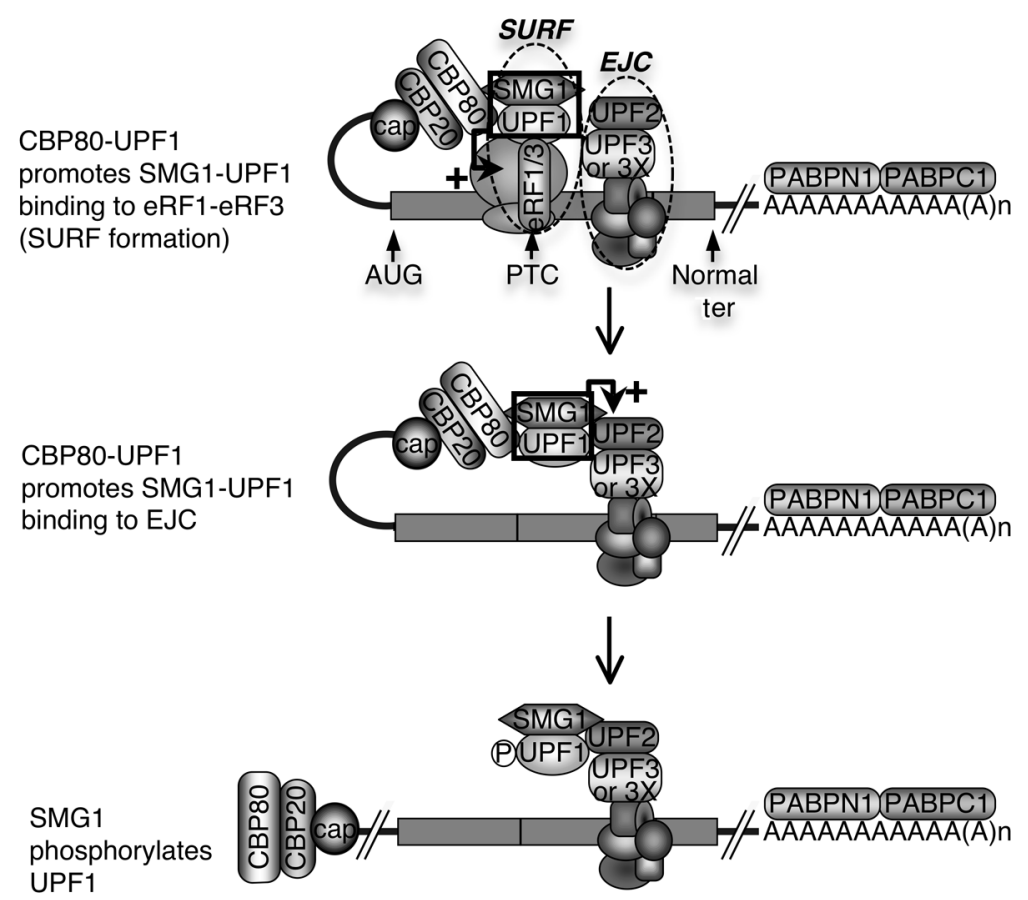

CBP80-UPF1 promotes SMG1-UPF1 binding to EJC

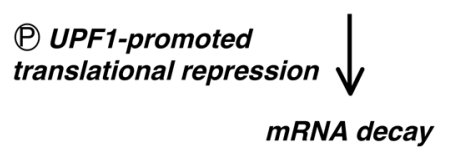

Figure 3. During NMD, interaction of CBP80 with the UPF1 promotes the joining of SMG1-UPF1 to eRF1-eRF3 at a PTC to form SURF and, subsequently, the joining of SMG1-UPF1 to a PTC-distal EJC. At the point when ribosomes engaged in the pioneer round of translation reach a PTC, CBP80 of CBC interacts directly but transiently or weakly with the NMD factor UPF1 (Hwang et al. 2010). It is unclear whether UPF1 is already associated with its kinase SMG1. Interaction of CBP80-UPF1 augments the binding of UPF1 and SMG1 to the heterodimer of eRF1 and eRF3 that is poised at the PTC. Binding forms the SURF complex. It is possible, but not certain, that the same UPF1 molecule that associates with CBP80 also ends up in SURF. Joining of SMG1-UPF1 to eRF1-eRF3 appears to be in competition with joining of poly(A)-tail-bound PABPC1 to eRF1-eRF3, the latter of which likely occurs predominantly at termination codons that do not trigger NMD (Ivanov et al. 2008; Singh et al. 2008). CBP80 subsequently promotes the joining of SMG1-UPF1 to a PTC-distal EJC, which generally consists of NMD factors UPF2 and either UPF3 or UPF3X. Contrary to some (Yamashita et al. 2009) but consistent with other (Kashima et al. 2006) published data, we found no convincing evidence that eRF1 or eRF3 also join the EJC, although we are able to detect an association of CBP80 with the EJC (Hwang et al. 2010). SMG1-UPF1 binding to the EJC triggers UPF1 phosphorylation (circled "P"), which inhibits further translation initiation events at the mRNA 5' end and enhances the efficiency of mRNA decay (Kashima et al. 2006; Isken et al. 2008). (AUG) translation initiation codon. (Normal ter) normal termination codon; not shown is PABPN1 at the poly(A) tail, which is present concomitantly with PABPC1.

tration of intact EJCs is reduced using either UPF2 or Y14 small interfering RNA (siRNA) (Kashima et al. 2006; H Sato and LE Maquat, unpubl.). Given that UPF1 and SMG1 do not detectably coimmunoprecipitate with eIF4E (Hosoda et al. 2005; Kashima et al. 2006), there must be a feature of the pioneer round of translation that promotes SURF complex formation on CBC-bound mRNAs to distinguish these mRNAs from their eIF4E-bound counterparts. We propose that this feature is CBP80, which appears to chaperone UPF1 together with SMG1 to eRF1 and eRF3 that are poised at a PTC. Chaperoning appears to allow the interaction of UPF1 with eRF3 at a PTC to outcompete the interaction of PABPC1 with eRF3 at a PTC, the latter of which occurs less efficiently than at a termination codon that does not trigger NMD (Ivanov et al. 2008; Singh et al. 2008). After SURF formation at a PTC, CBP80 chaperones SMG1 and UPF1, presumably from SURF, to a PTC-distal EJC. Binding generally in- volves a direct interaction of UPF1 with the EJC constituent UPF2 because a UPF1 variant that cannot bind UPF2 associates abnormally efficiently with eRF1 and eRF3 (Kashima et al. 2006). Competition between eRF3 and UPF2 for binding to UPF1 has never been demonstrated, although both proteins bind to the UPF1 cysteinehistidine-rich region (Ivanov et al. 2008; Clerici et al. 2009; Gong et al. 2009). However, our data are consistent with the possibility of competition: The only SURF constituents that we detect binding to an EJC are SMG1 and UPF1, and they bind along with CBP80.

UPF1 and SMG1 binding to an EJC may involve a direct interaction of not only UPF1, but also SMG1 with UPF2 based on the finding that the FLAG-tagged carboxy-terminal domain of SMG1 purified from mammalian cells interacts with in vitro-synthesized UPF2 (Kashima et al. 2006). Furthermore, Y14 coimmunoprecipitates with UPF2 and SMG1 in the presence of RNase 
A (Kashima et al. 2006). UPF1 and SMG1 binding to an EJC triggers the SMG1-mediated phosphorylation of UPF1 (Ohnishi et al. 2003; Kashima et al. 2006; Wittmann et al. 2006). Consequently, the joining of $60 \mathrm{~S}$ ribosomal subunits to the $43 \mathrm{~S}$ preinitiation complex that is poised at the translation initiation codon of the NMD target is inhibited by the binding of phosphorylated UPF1 to the eIF3 constituent of the preinitiation complex (Isken et al. 2008). Remarkably, this mechanism of translation repression appears to be essential for mRNA decay, which involves decapping, 5'-to-3' exonuclease, deadenylating, and 3'-to-5' exonuclease activities (Lykke-Andersen 2002; Lejeune et al. 2003; Lehner and Sanderson 2004; Unterholzner and Izaurralde 2004; Yamashita et al. 2005) as well as endonucleolytic activity (Huntzinger et al. 2008; Eberle et al. 2009).

\section{CONCLUSIONS}

mRNAs are generally associated with RNA-binding proteins and noncoding RNAs. Some binding proteins typify all mRNPs. As one example, CBC is thought to bind to the $5^{\prime}$-cap structures of all functional newly synthesized mRNAs. As another example, EJCs are believed to associate with the exon-exon junctions of all newly synthesized spliced mRNAs, although data indicate that EJCs are neither static nor homogenous in composition (see, e.g., Viegas et al. 2007; Zhang and Krainer 2007). Other binding proteins and noncoding RNAs characterize only a subset of mRNPs and often under only particular conditions. For example, the AU-rich element of tumor necrosis factor (TNF) $\alpha$ mRNA binds the fragile-X mental retardation-related protein 1 , Argonaute 1 protein, and microRNA (miRNA) miR369-3p when cells are serum deprived to activate TNF $\alpha$ mRNA translation (Steitz and Vasudevan 2009).

A number of protein-protein interactions that have been reported to occur within cellular mRNPs span a sufficient number of mRNA nucleotides to result in mRNA looping. As reviewed here, data suggest that SURF complex formation at a PTC and the subsequent binding of SMG1 and UPF1, presumably from SURF, to a PTC-distal EJC involves cap-bound CBP80 interactions with UPF1 in SURF and, subsequently, UPF1 at the EJC, all via mRNA looping. Likewise, models for how PABP-interacting protein 2 mediates the translational repression of eIF4E-bound mRNAs that are polyadenylated, or how GW182 mediates that the translational repression of eIF4E-bound mRNAs that are not only polyadenylated but also targeted by a miRNA, imply that PABPC1 at an mRNA 3' end forms a complex with eIF4G at an mRNA $5^{\prime}$ end (Derry et al. 2006; Tritschler et al. 2010). Similarly, the stem-loop-binding protein (SLBP)-interacting protein 1 appears to bridge the SLBP at the $3^{\prime}$ end of replication-dependent histone mRNAs, which lack a poly(A) tail, and eIF4G at the mRNA 5' end (Cakmakci et al. 2008). Many other examples derive from developmentally regulated interactions between a sequence-specific 3'UTR-binding protein and cap-associated eIF4E via a bridging protein that represses translation initiation (Jackson et al. 2010).
Viral mRNAs also appear to form loops by virtue of interactions among their associated proteins. For example, rotavirus nonstructural protein 3 , which is functionally orthologous to PABP, binds the $3^{\prime}$ end of viral mRNAs, which are capped but not polyadenylated, as well as eIF4G (Groft and Burley 2002). As another example, PABP at the poly(A) tail of genomic poliovirus RNA, which is not capped, interacts with a cellular poly $(\mathrm{C})$-binding protein that associates with the $5^{\prime}$-end cloverleaf structure (Herold and Andino 2001). mRNA looping can also occur via base pairing between $3^{\prime}$ and $5^{\prime}$ sequences to form a kissing stem loop in a way that may be stabilized by proteins, as evidenced by barley yellow dwarf luteovirus transcripts as well as transcripts from the related soybean dwarf virus and the unrelated tobacco necrosis virus, all of which are neither capped nor polyadenylated (Guo et al. 2001).

Future studies will undoubtedly provide additional insight into the dynamics of mRNP structure and their contributions to mRNA function.

\section{ACKNOWLEDGMENTS}

This work was supported by the National Institutes of Health grants R01 GM59614 and GM59614-11S to L.E.M. H.S. is supported in part by a Fellowship from the Japan Society for the Promotion of Science.

\section{REFERENCES}

Afonina E, Stauber R, Pavlakis GN. 1998. The human poly(A)binding protein 1 shuttles between the nucleus and the cytoplasm. J Biol Chem 273: 13015-13021.

Bear DG, Fomproix N, Soop T, Bjorkroth B, Masich S, Daneholt B. 2003. Nuclear poly(A)-binding protein PABPN1 is associated with RNA polymerase II during transcription and accompanies the released transcript to the nuclear pore. Exp Cell Res 286: 332-344.

Cakmakçi NG, Lerner RS, Wagner EJ, Zheng L, Marzluff WF. 2008. SLIP1, a factor required for activation of histone mRNA translation by the stem-loop binding protein. Mol Cell Biol 28: 1182-1194.

Calero G, Wilson KF, Ly T, Rios-Steiner JL, Clardy JC, Cerione RA. 2002. Structural basis of $m^{7} \mathrm{GpppG}$ binding to the nuclear cap-binding protein complex. Nat Struct Biol 9: 912-917.

Chamieh H, Ballut L, Bonneau F, Le Hir H. 2008. NMD factors UPF2 and UPF3 bridge UPF1 to the exon junction complex and stimulate its RNA helicase activity. Nat Struct Mol Biol 15: 8593.

Cheng H, Dufu K, Lee CS, Hsu JL, Dias A, Reed R. 2006. Human mRNA export machinery recruited to the $5^{\prime}$ end of mRNA. Cell 127: 1389-1400.

Clerici M, Mourão A, Gutsche I, Gehring NH, Hentze MW, Kulozik A, Kadlec J, Sattler M, Cusack S. 2009. Unusual bipartite mode of interaction between the nonsense-mediated decay factors, UPF1 and UPF2. EMBO J 28: 2293-2306.

Chiu SY, Lejeune F, Ranganathan AC, Maquat LE. 2004. The pioneer translation initiation complex is functionally distinct from but structurally overlaps with the steady-state translation initiation complex. Genes Dev 18: 745-754.

Derry MC, Yanagiya A, Martineau Y, Sonenberg N. 2006. Regulation of poly(A)-binding protein through PABP-interacting proteins. Cold Spring Harb Symp Quant Biol 71: 537-543.

Dias SM, Wilson KF, Rojas KS, Ambrosio AL, Cerione RA. 2009. The molecular basis for the regulation of the cap-binding complex by the importins. Nat Struct Mol Biol 16: 930-937.

Dostie J, Dreyfuss G. 2002. Translation is required to remove Y14 from mRNAs in the cytoplasm. Curr Biol 12: 1060-1067. 
Eberle AB, Lykke-Andersen S, Mühlemann O, Jensen TH. 2009. SMG6 promotes endonucleolytic cleavage of nonsense mRNA in human cells. Nat Struct Mol Biol 16: 49-55.

Fortes P, Inada T, Preiss T, Hentze MW, Mattaj IW, Sachs AB. 2000. The yeast nuclear cap binding complex can interact with translation factor eIF4G and mediate translation initiation. Mol Cell 6: 191-196.

Franks TM, Singh G, Lykke-Andersen J. 2010. Upf1 ATPase-dependent mRNP disassembly is required for completion of nonsense-mediated mRNA decay. Cell 143: 938-950.

Gehring NH, Lamprinaki S, Kulozik AE, Hentze MW. 2009. Disassembly of exon junction complexes by PYM. Cell 137: 536548.

Gong C, Kim YK, Woeller CF, Tang Y, Maquat LE. 2009. SMD and NMD are competitive pathways that contribute to myogenesis: Effects on PAX3 and myogenin mRNAs. Genes Dev 23: 54-66.

Görlich D, Kraft R, Kostka S, Vogel F, Hartmann E, Laskey RA, Mattaj IW, Izaurralde E. 1996. Importin provides a link between nuclear protein import and U snRNA export. Cell 87: 21-32.

Groft CM, Burley SK. 2002. Recognition of eIF4G by rotavirus NSP3 reveals a basis for mRNA circularization. Mol Cell 9: 1273-1283.

Gross JD, Moerke NJ, von der Haar T, Lugovskoy AA, Sachs AB, McCarthy JE, Wagner G. 2003. Ribosome loading onto the mRNA cap is driven by conformational coupling between eIF4G and eIF4E. Cell 115: 739-750.

Guo L, Allen EM, Miller WA. 2001. Base-pairing between untranslated regions facilitates translation of uncapped, nonpolyadenylated viral RNA. Mol Cell 7: 1103-1109.

Haghighat A, Sonenberg N. 1997. eIF4G dramatically enhances the binding of eIF4E to the mRNA 5'-cap structure. J Biol Chem 272: $21677-21680$

Herold J, Andino R. 2001. Poliovirus RNA replication requires genome circularization through a protein-protein bridge. Mol Cell 7: 581-591

Hoshino S, Imai M, Kobayashi T, Uchida N, Katada T. 1999. The eukaryotic polypeptide chain releasing factor (eRF3/GSPT) carrying the translation termination signal to the 3'-poly(A) tail of mRNA. Direct association of eRF3/GSPT with polyadenylatebinding protein. $J$ Biol Chem 274: 16677-16680.

Hosoda N, Kim YK, Lejeune F, Maquat LE. 2005. CBP80 promotes interaction of Upf1 with Upf2 during nonsense-mediated mRNA decay in mammalian cells. Nat Struct Mol Biol 12: 893-901.

Hosoda N, Lejeune F, Maquat LE. 2006. Evidence that poly(A) binding protein $\mathrm{C} 1$ binds nuclear pre-mRNA poly(A) tails. Mol Cell Biol 26: 3085-3097.

Huntzinger E, Kashima I, Fauser M, Saulière J, Izaurralde E. 2008. SMG6 is the catalytic endonuclease that cleaves mRNAs containing nonsense codons in metazoan. RNA 14: 2609-2617.

Hwang J, Sato H, Matsuda D, Tang Y, Maquat LE. 2010. UPF1 association with the cap-binding protein, CBP80, promotes nonsense-mediated mRNA decay at two distinct steps. Mol Cell 39: 396-409.

Ishigaki Y, Li X, Serin G, Maquat LE. 2001. Evidence for a pioneer round of mRNA translation: mRNAs subject to nonsense-mediated decay in mammalian cells are bound by CBP80 and CBP20. Cell 106: 607-617.

Isken O, Maquat LE. 2007. Quality control of eukaryotic mRNA: Safeguarding cells from abnormal mRNA function. Genes Dev 21: $1833-1856$.

Isken O, Maquat LE. 2008. The multiple lives of NMD factors: Balancing roles in gene and genome regulation. Nat Rev Genet 9: 699-712.

Isken O, Kim YK, Hosoda N, Mayeur GL, Hershey JW, Maquat LE. 2008. Upf1 phosphorylation triggers translational repression during nonsense-mediated mRNA decay. Cell 133: 314-327.

Ivanov PV, Gehring NH, Kunz JB, Hentze MW, Kulozik AE. 2008. Interactions between UPF1, eRFs, PABP and the exon junction complex suggest an integrated model for mammalian NMD pathways. EMBO J 27: 736-747.

Izaurralde E, Lewis J, McGuigan C, Jankowska M, Darzynkiewicz E, Mattaj IW. 1994. A nuclear cap binding protein complex in- volved in pre-mRNA splicing. Cell 78: 657-668.

Jackson RJ, Hellen CU, Pestova TV. 2010. The mechanism of eukaryotic translation initiation and principles of its regulation. Nat Rev Mol Cell Biol 11: 113-127.

Kashima I, Yamashita A, Izumi N, Kataoka N, Morishita R, Hoshino S, Ohno M, Dreyfuss G, Ohno S. 2006. Binding of a novel SMG-1-Upf1-eRF1-eRF3 complex (SURF) to the exon junction complex triggers Upfl phosphorylation and nonsensemediated mRNA decay. Genes Dev 20: 355-367.

Kim VN, Kataoka N, Dreyfuss G. 2001. Role of the nonsense-mediated decay factor hUpf3 in the splicing-dependent exon-exon junction complex. Science 293: 1832-1836.

Kühn U, Wahle E. 2004. Structure and function of poly(A) binding proteins. Biochim Biophys Acta 1678: 67-84.

Le Hir H, Andersen GR. 2008. Structural insights into the exon junction complex. Curr Opin Struct Biol 18: 112-119.

Lehner B, Sanderson CM. 2004. A protein interaction framework for human mRNA degradation. Genome Res 14: 1315-1323.

Lejeune F, Ishigaki Y, Li X, Maquat LE. 2002. The exon junction complex is detected on CBP80-bound but not eIF4E-bound mRNA in mammalian cells: Dynamics of mRNP remodeling. EMBO J 21: 3536-3545.

Lejeune F, Li X, Maquat LE. 2003. Nonsense-mediated mRNA decay in mammalian cells involves decapping, deadenylating, and exonucleolytic activities. Mol Cell 12: 675-687.

Lejeune F, Ranganathan AC, Maquat LE. 2004. eIF4G is required for the pioneer round of translation in mammalian cells. Nat Struct Mol Biol 11: 992-1000.

Lewis JD, Izaurralde E. 1997. The role of the cap structure in RNA processing and nuclear export. Eur J Biochem 247: 461-469.

Lykke-Andersen J. 2002. Identification of a human decapping complex associated with hUpf proteins in nonsense-mediated decay. Mol Cell Biol 22: 8114-8121.

Lykke-Andersen J, Shu MD, Steitz JA. 2000. Human Upf proteins target an mRNA for nonsense-mediated decay when bound downstream of a termination codon. Cell 103: 1121-1131.

Lykke-Andersen J, Shu MD, Steitz JA. 2001. Communication of the position of exon-exon junctions to the mRNA surveillance machinery by the protein RNPS1. Science 293: 1836-1839.

Maquat LE, Tarn WY, Isken O. 2010. The pioneer round of translation: Features and functions. Cell 142: 368-374.

Marcotrigiano J, Gingras AC, Sonenberg N, Burley SK. 1997. Cocrystal structure of the messenger RNA $5^{\prime}$ cap-binding protein (eIF4E) bound to 7-methyl-GDP. Cell 89: 951-961.

Matsuo H, Li H, McGuire AM, Fletcher CM, Gingras AC, Sonenberg N, Wagner G. 1997. Structure of translation factor eIF4E bound to $\mathrm{m}^{7} \mathrm{GDP}$ and interaction with 4E-binding protein. Nat Struct Biol 4: 717-724.

Mazza C, Ohno M, Segref A, Mattaj IW, Cusack S. 2001. Crystal structure of the human nuclear cap binding complex. Mol Cell 8: $383-396$.

Mazza C, Segref A, Mattaj IW, Cusack S. 2002. Large-scale induced fit recognition of an $m^{7} \mathrm{GpppG}$ cap analogue by the human nuclear cap-binding complex. EMBO J 21: 5548-5557.

McKendrick L, Thompson E, Ferreira J, Morley SJ, Lewis JD. 2001. Interaction of eukaryotic translation initiation factor $4 \mathrm{G}$ with the nuclear cap-binding complex provides a link between nuclear and cytoplasmic functions of the $\mathrm{m}^{7}$ guanosine cap. Mol Cell Biol 21: 3632-3641.

Niedzwiecka A, Stepinski J, Darzynkiewicz E, Sonenberg N, Stolarski R. 2002. Positive heat capacity change upon specific binding of translation initiation factor eIF4E to mRNA 5' cap. Biochemistry 41: 12140-12148.

Nicholson P, Yepiskoposyan H, Metze S, Orozco RZ, Kleinschmidt N, Mühlemann O. 2010. Nonsense-mediated mRNA decay in human cells: Mechanistic insights, functions beyond quality control and the double-life of NMD factors. Cell Mol Life Sci 67: 677-700.

Ohnishi T, Yamashita A, Kashima I, Schell T, Anders KR, Grimson A, Hachiya T, Hentze MW, Anderson P, Ohno S. 2003. Phosphorylation of hUPF1 induces formation of mRNA surveillance complexes containing hSMG-5 and hSMG-7. Mol Cell 12: $1187-1200$. 
Rebbapragada I, Lykke-Andersen J. 2009. Execution of nonsensemediated mRNA decay: What defines a substrate? Curr Opin Cell Biol 21: 394-402.

Sato H, Maquat LE. 2009. Remodeling of the pioneer translation initiation complex involves translation and the karyopherin importin $\beta$. Genes Dev 23: 2537-2550.

Sato H, Hosoda N, Maquat LE. 2008. Efficiency of the pioneer round of translation affects the cellular site of nonsense-mediated mRNA decay. Mol Cell 29: 255-262.

Silva AL, Romão L. 2009. The mammalian nonsense-mediated mRNA decay pathway: To decay or not to decay! Which players make the decision? FEBS Lett 583: 499-505.

Singh G, Jakob S, Kleedehn MG, Lykke-Andersen J. 2007. Communication with the exon-junction complex and activation of nonsense-mediated mRNA decay by human Upf proteins occur in the cytoplasm. Mol Cell 27: 780-792.

Singh G, Rebbapragada I, Lykke-Andersen J. 2008. A competition between stimulators and antagonists of Upf complex recruitment governs human nonsense-mediated mRNA decay. PLoS Biol 6: 860-871.

Sonenberg N, Hinnebusch AG. 2009. Regulation of translation initiation in eukaryotes: Mechanisms and biological targets. Cell 136: 731-745.

Steitz JA, Vasudevan S. 2009. miRNPs: Versatile regulators of gene expression in vertebrate cells. Biochem Soc Trans 37: 931-935.

Stephenson LS, Maquat LE. 1996. Cytoplasmic mRNA for human triosephosphate isomerase is immune to nonsense-mediated decay despite forming polysomes. Biochimie 78: 1043-1047.

Tomoo K, Shen X, Okabe K, Nozoe Y, Fukuhara S, Morino S, Ishida T, Taniguchi T, Hasegawa H, Terashima A, et al. 2002. Crystal structures of 7-methylguanosine 5'-triphosphate $\left(\mathrm{m}^{7} \mathrm{GTP}\right)$ - and $\mathrm{P}^{1}$-7 -methylguanosine- $\mathrm{P}^{3}$-adenosine- $5^{\prime}, 5^{\prime}$-triphosphate $\left(\mathrm{m}^{7} \mathrm{GpppA}\right)$-bound human full-length eukaryotic initiation factor 4E: Biological importance of the C-terminal flexible region. Biochem J 362: 539-544.

Tran EJ, Zhou Y, Corbett AH, Wente SR. 2007. The DEAD-box protein Dbp5 controls mRNA export by triggering specific RNA:protein remodeling events. Mol Cell 28: 850-859.
Tritschler F, Huntzinger E, Izaurralde E. 2010. Role of GW182 proteins and PABPC1 in the miRNA pathway: A sense of déjà vu. Nat Rev Mol Cell Biol 11: 379-384.

Unterholzner L, Izaurralde E. 2004. SMG7 acts as a molecular link between mRNA surveillance and mRNA decay. Mol Cell 16: $587-596$.

Viegas MH, Gehring NH, Breit S, Hentze MW, Kulozik AE. 2007. The abundance of RNPS1, a protein component of the exon junction complex, can determine the variability in efficiency of the nonsense-mediated decay pathway. Nucleic Acids Res 35: 4542-4551.

Visa N, Izaurralde E, Ferreira J, Daneholt B, Mattaj IW. 1996. A nuclear cap-binding complex binds Balbiani ring pre-mRNA cotranscriptionally and accompanies the ribonucleoprotein particle during nuclear export. J Cell Biol 133: 5-14.

Wen Y, Shatkin AJ. 2000. Cap methyltransferase selective binding and methylation of GpppG-RNA are stimulated by importin- $\alpha$. Genes Dev 14: 2944-2949.

Wittmann J, Hol EM, Jack HM. 2006. hUPF2 silencing identifies physiologic substrates of mammalian nonsense-mediated mRNA decay. Mol Cell Biol 26: 1272-1287.

Worch R, Niedzwiecka A, Stepinski J, Mazza C, JankowskaAnyszka M, Darzynkiewicz E, Cusack S, Stolarski R. 2005. Specificity of recognition of mRNA $5^{\prime}$ cap by human nuclear cap-binding complex. RNA 11: 1355-1363.

Yamashita A, Chang TC, Yamashita Y, Zhu W, Zhong Z, Chen CY, Shyu AB. 2005. Concerted action of poly(A) nucleases and decapping enzyme in mammalian mRNA turnover. Nat Struct Mol Biol 12: 1054-1063.

Yamashita A, Izumi N, Kashima I, Ohnishi T, Saari B, Katsuhata Y, Muramatsu R, Morita T, Iwamatsu A, Hachiya T, et al. 2009. SMG-8 and SMG-9, two novel subunits of the SMG-1 complex, regulate remodeling of the mRNA surveillance complex during nonsense-mediated mRNA decay. Genes Dev 23: 1091-1105.

Zhang Z, Krainer AR. 2007. Splicing remodels messenger ribonucleoprotein architecture via eIF4A3-dependent and -independent recruitment of exon junction complex components. Proc Natl Acad Sci 104: 11574-11579. 


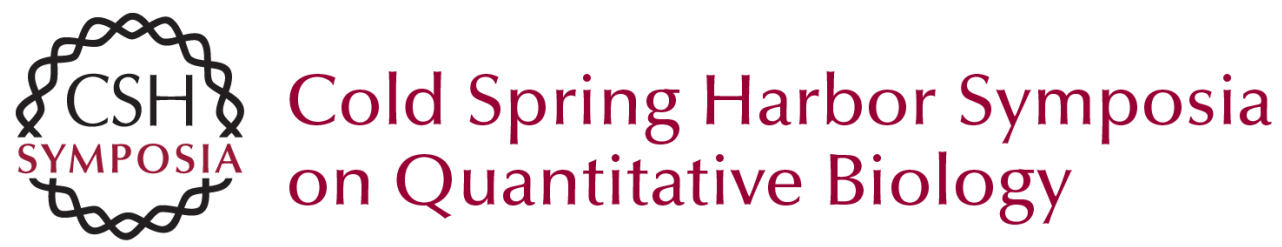

\title{
CBP80-Promoted mRNP Rearrangements during the Pioneer Round of Translation, Nonsense-Mediated mRNA Decay, and Thereafter
}

\author{
L.E. Maquat, J. Hwang, H. Sato, et al.
}

Cold Spring Harb Symp Quant Biol 2010 75: 127-134 originally published online March 29, 2011 Access the most recent version at doi:10.1101/sqb.2010.75.028

References This article cites 76 articles, 25 of which can be accessed free at: http://symposium.cshlp.org/content/75/127.full.html\#ref-list-1

\section{License}

Email Alerting

Receive free email alerts when new articles cite this article - sign up in the box at the Service top right corner of the article or click here.

To subscribe to Cold Spring Harbor Symposia on Quantitative Biology go to: http://symposium.cshlp.org/subscriptions 